\title{
Measurement report: Intra-, inter-annual variability and source apportionment of VOCs during 2018-2020 in Zhengzhou,
} Central China

\author{
Shijie Yu ${ }^{a, b}$, Shenbo Wang ${ }^{b, c}$, Ruixin Xu ${ }^{b, c}$, Dong Zhang a,b, Meng Zhang ${ }^{\mathrm{e}}$, Fangcheng $\mathrm{Su}^{\mathrm{b}, \mathrm{c}}$, \\ Ruiqin Zhang ${ }^{b, c *}$, Lingling Wang ${ }^{d *}$ \\ a. College of Chemistry, Zhengzhou University, Zhengzhou 450001, China \\ b. Institute of Environmental Sciences, Zhengzhou University, Zhengzhou \\ 450001, China \\ c. School of Ecology and Environment, Zhengzhou University, Zhengzhou \\ 450001, China \\ d. Environmental Monitoring Center of Henan Province, Zhengzhou 450000 \\ e. Pingdingshan Ecological Environment Monitoring Center of Henan Province, \\ Pingdingshan 467000, China
}

*Correspondence author. Research Institute of Environmental Science, College of Environment and Ecology, Zhengzhou University High-tech Development Zone, Zhengzhou, Henan, PR China, 450001

E-mail address: rqzhang@,zzu.edu.cn

*Correspondence author. Environmental Monitoring Center of Henan Province, 
Zhengzhou, Henan, PR China, 450000

E-mail address: Lindaw2007@,126.com

\section{Supplementary Materials:}

Text S1 Positive Matrix Factorization model

Text S2 Relative reactivity of VOCs

\section{Table list:}

Table S1. Results of monitored 57 VOCs species monitored: MDLs, concentrations with statistical analysis (unit: ppbv) (during 2018-2020).

Table S2 Average ambient VOCs concentrations and chemical species during 2018 to 2020 .

Table S3 Variations in the monthly average of meteorological parameters (T, RH, UV, and WS $)$ and pollutant gases $\left(\mathrm{O}_{3}, \mathrm{NO}_{2}, \mathrm{CO}\right.$, and TVOC).

Table S4 The detailed contribution of each VOC group to the total $\mathrm{OH}$ reactivity during the sampling periods.

Table S5 The detailed contribution of each VOC group to the total $\mathrm{OH}$ reactivity in different seasons.

\section{Figure list:}

Fig. S1 The ratios of Q/Qexp at factor size ranged from 2-8.

Fig. S2 Monthly changes in the concentrations of VOCs in Zhengzhou.

Fig. S3 Diurnal variations of VOCs meteorological conditions during the measurements.

Fig. S4 Seasonal variation in B/T and i-/n-Pentane in Zhengzhou.

Fig. S5 Comparison of the relative contributions of $\mathrm{OH}, \mathrm{O}_{3}$ and $\mathrm{NO}_{3}$ of the $\mathrm{AOC}$ in Zhengzhou in different seasons.

Fig. S6 Box and whisker plots of VOC profiles based on different scales during the 
entire sampling period.

Fig.S7 Contribution of each source calculated using PMF, OFP, and PE. 


\section{Text S1 Positive Matrix Factorization model}

The source of VOCs was performed with the EPA PMF 5.0 model, which is a widely receptor model for source apportionment (Gao et al., 2018; Yadav et al., 2019). Detailed information about this method was described in the user manual (Norris et al., 2014) and related literatures (Song et al., 2019a, 2019b). It should be noted that not all of the VOC species were used in the PMF analysis. In this study, the principles for the selection of VOC species are established based on previous studies and listed as follows. (1) species with more than $25 \%$ data lacking or that fell below the MDLs were rejected, which follows the methodology of previous studies (Zhou et al., 2019); (2) species with short atmospheric lifetimes were excluded because they rapidly react away in the atmosphere; and (3) species with represented source tracers of emission sources were retained (For example, in the case of isoprene). Eventually, a total of 38 VOC species were selected for source apportionment analysis. In this study, seven-factors are extracted by PMF model based on: (1) principal component analysis of the VOC data; (2) VOC emission inventory of research region based on field investigation; and (3) Q/Qexp ratio for different factor numbers in the PMF (Fig. S1). 


\section{Text S2 Relative reactivity of VOCs}

To better understand the role of VOCs in the formation of troposphere $\mathrm{O}_{3}$, reactive substances should be firstly determined. In this paper, OFP and the PE concentration are investigated to analyze the chemical reactivity of VOC species.

OFP is based on the concentration and MIR of each VOC species, as given in the following equation:

$$
\operatorname{OFP}_{(i)}=\text { concentration }_{(i)} \times \operatorname{MIR}(i) \times \frac{M_{(i)}}{M_{(\text {Ozone })}}
$$

Where $\mathrm{M}($ ozone $)$ and $\mathrm{M}(\mathrm{i})$ are the molar mass of $\mathrm{O}_{3}$ and individual VOC species, respectively. $\mathrm{OFP}_{(\mathrm{i})}$ is the $\mathrm{O}_{3}$ formation potential of VOC species $\mathrm{i}$ and concentration (VOCs) is the mass concentration of each VOC $\left(\mu \mathrm{g} \mathrm{m}^{-3}\right)$. And the MIR value of each VOC species was given by Carter (1994).

The PE concentration is defined as Eq. (2)

$$
\mathrm{PE}_{(\mathrm{i})}=\text { concentration }_{(\mathrm{i})} \times \frac{\mathrm{k}_{\mathrm{OH}}(\mathrm{i})}{\mathrm{k}_{\mathrm{OH}}(\text { propene })}
$$

Where $\mathrm{k}_{\mathrm{OH}(\mathrm{i})}$ and $\mathrm{kOH}_{\mathrm{OH}}$ (propene) represent a rate constant for the reactivity of each VOC with an $\mathrm{OH}$ radical and the reaction of $\mathrm{C}_{3} \mathrm{H}_{6}$ with $\mathrm{OH}$ radicals. The kOH rate constants were from Atkinson and Arey (2003). 
Table S1. Results of monitored 57 VOCs species monitored: MDLs, concentrations with statistical analysis (unit: ppbv) (during 2018-2020).

\begin{tabular}{|c|c|c|c|}
\hline & MDL & Mean & SD \\
\hline Ethane & 0.08 & 8.8 & 5 \\
\hline Ethene & 0.07 & 4.7 & 4 \\
\hline Propane & 0.04 & 4.2 & 2.5 \\
\hline Acetylene & 0.04 & 2.6 & 3.3 \\
\hline n-Butane & 0.03 & 2.3 & 1.7 \\
\hline i-Pentane & 0.02 & 1.9 & 2 \\
\hline Toluene & 0.03 & 1.6 & 1.1 \\
\hline i-Butane & 0.03 & 1.3 & 1 \\
\hline n-Pentane & 0.02 & 1.2 & 1.1 \\
\hline Benzene & 0.03 & 1.1 & 0.6 \\
\hline m p-Xylene & 0.02 & 1 & 1 \\
\hline Cyclopentane & 0.02 & 0.7 & 1.1 \\
\hline Propene & 0.03 & 0.7 & 1.1 \\
\hline 1-Isoprene & 0.02 & 0.6 & 0.9 \\
\hline Ethylbenzene & 0.02 & 0.5 & 0.4 \\
\hline 3-Methylpentane & 0.04 & 0.4 & 0.4 \\
\hline n-Hexane & 0.04 & 0.4 & 0.5 \\
\hline o-Xylene & 0.02 & 0.3 & 0.3 \\
\hline Styrene & 0.02 & 0.3 & 0.3 \\
\hline 1-Butene & 0.02 & 0.2 & 0.3 \\
\hline 2-Methylpentane & 0.03 & 0.2 & 0.3 \\
\hline cis-2-Butene & 0.03 & 0.2 & 0.4 \\
\hline m-Ethyltoluene & 0.04 & 0.2 & 0.2 \\
\hline n-Heptane & 0.03 & 0.2 & 0.3 \\
\hline p-Diethylbenzene & 0.02 & 0.2 & 0.2 \\
\hline trans-2-Butene & 0.02 & 0.2 & 0.6 \\
\hline 1,2,3-Trimethylbenzene & 0.02 & 0.1 & 0.1 \\
\hline $1,2,4$-Trimethylbenzene & 0.12 & 0.1 & 0.1 \\
\hline 1,3,5-Trimethylbenzene & 0.03 & 0.1 & 0.1 \\
\hline 1-Hexene & 0.04 & 0.1 & 0.2 \\
\hline 1-Pentene & 0.03 & 0.1 & 0.1 \\
\hline 2,2,4-Trimethylpentane & 0.03 & 0.1 & 0.1 \\
\hline 2,2-Dimethylbutane & 0.04 & 0.1 & 0.1 \\
\hline 2,3-Dimethylbutane & 0.03 & 0.1 & 0.1 \\
\hline 2,4-Dimethylpentane & 0.03 & 0.1 & 0.1 \\
\hline 2-Methylheptane & 0.02 & 0.1 & 0.2 \\
\hline 2-Methylhexane & 0.03 & 0.1 & 0.1 \\
\hline 3-Methylhexane & 0.03 & 0.1 & 0.1 \\
\hline cis-2-Pentene & 0.03 & 0.1 & 0.2 \\
\hline Cyclohexane & 0.04 & 0.1 & 0.1 \\
\hline i-Propylbenzene & 0.02 & 0.1 & 0.1 \\
\hline
\end{tabular}


Methylcyclohexane

0.03

0.04

0.1

0.1

Methylcyclopentane

0.06

0.1

0.2

n-Decane

0.03

0.1

0.1

n-Dodecane

0.1

0.2

n-Nonane

0.02

0.1

0.2

n-Octane

0.02

n-Propylbenzene

0.02

n-Undecane

0.02

o-Ethyltoluene

0.02

p-Ethyltoluene

0.04

0.03

trans-2-Pentene

0.02

0.03

2,3-Dimethylpentane

0.02

0.1

0.2

0.1

0.2

3-Methylheptane

0.02

0.1

0.3

m-Diethylbenzene

$0.1 \quad 0.1$

$0.1 \quad 0.1$

$\begin{array}{ll}0.1 & 0.2\end{array}$

BDL

BDL

BDL

BDL 
Table S2 Average ambient VOCs concentrations and chemical species during 2018 to 2020.

\begin{tabular}{cccccc}
\hline & Alkanes & Alkenes & Alkynes & Aromatics & TVOC \\
\hline 2018 & $25.2 \pm 17.2$ & $9.2 \pm 5.7$ & $4.2 \pm 4.3$ & $6.4 \pm 2.9$ & $45.0 \pm 25.2$ \\
2019 & $23.5 \pm 13.9$ & $5.2 \pm 5.1$ & $2.9 \pm 4.1$ & $5.1 \pm 3$ & $36.7 \pm 22$ \\
2020 & $19.4 \pm 9.9$ & $5.5 \pm 4.0$ & $0.7 \pm 0.8$ & $4.9 \pm 3.1$ & $30.5 \pm 15.4$ \\
Average & $23 \pm 19.5$ & $7.1 \pm 3.3$ & $2.6 \pm 2.6$ & $5.5 \pm 1.3$ & $38.2 \pm 15.6$ \\
\hline
\end{tabular}


Table S3 Variations in the monthly average of meteorological parameters ( $\mathrm{T}, \mathrm{RH}, \mathrm{UV}$, and WS $)$ and pollutant gases $\left(\mathrm{O}_{3}, \mathrm{NO}_{2}, \mathrm{CO}\right.$, and TVOC).

\begin{tabular}{cccccccccc}
\hline Month & $\mathrm{RH}$ & $\mathrm{Pr}$ & $\mathrm{T}$ & $\mathrm{WS}$ & $\mathrm{UV}$ & $\mathrm{TVOC}$ & $\mathrm{NO}_{2}$ & $\mathrm{O}_{3}$ & $\mathrm{CO}$ \\
\hline 1 & $46.4 \pm 22$ & $7 \pm 0.1$ & $3.1 \pm 3.3$ & $1.3 \pm 0.8$ & $85 \pm 33.8$ & $62.6 \pm 32.2$ & $65.4 \pm 29.4$ & $22.6 \pm 19.5$ & $1.4 \pm 0.7$ \\
2 & $55.9 \pm 16.1$ & $3.9 \pm 0$ & $4.3 \pm 5$ & $1.2 \pm 1$ & $114.5 \pm 45.3$ & $45.5 \pm 26$ & $45.3 \pm 29.5$ & $50.4 \pm 35.5$ & $1.2 \pm 0.6$ \\
3 & $38.1 \pm 16.7$ & $3.3 \pm 0.1$ & $13.9 \pm 4.8$ & $1.6 \pm 0.8$ & $206.5 \pm 55$ & $40 \pm 18.1$ & $48.4 \pm 29.3$ & $65.2 \pm 42.8$ & $0.5 \pm 0.3$ \\
4 & $52.2 \pm 19.7$ & $20.2 \pm 0.2$ & $16.7 \pm 5.3$ & $1.9 \pm 1.1$ & $238.5 \pm 86.6$ & $47.5 \pm 20.6$ & $41.4 \pm 23.6$ & $76.7 \pm 49.1$ & $0.8 \pm 0.4$ \\
5 & $40.9 \pm 17.6$ & $0.2 \pm 0$ & $24.8 \pm 5.5$ & $1.5 \pm 0.9$ & $315.2 \pm 62.6$ & $26.7 \pm 12.5$ & $39 \pm 29.4$ & $100.1 \pm 62.1$ & $0.7 \pm 0.3$ \\
6 & $48.4 \pm 22.3$ & $20.6 \pm 0.2$ & $30 \pm 7.6$ & $0.6 \pm 0.4$ & $291.6 \pm 112$ & $26.3 \pm 10.9$ & $32.9 \pm 23.1$ & $114 \pm 63.9$ & $0.6 \pm 0.3$ \\
7 & $60 \pm 15.3$ & $18.2 \pm 0.3$ & $30.6 \pm 4.6$ & $0.4 \pm 0.2$ & $305.6 \pm 70.3$ & $27.6 \pm 13.1$ & $36.4 \pm 27.8$ & $110.4 \pm 66.9$ & $0.7 \pm 0.3$ \\
8 & $70 \pm 18$ & $60.5 \pm 0.6$ & $27.8 \pm 3.7$ & $0.4 \pm 0.2$ & $265 \pm 80.1$ & $21.5 \pm 8$ & $32.6 \pm 20.6$ & $95.5 \pm 58.5$ & $0.8 \pm 0.3$ \\
9 & $65 \pm 19.2$ & $1.9 \pm 0$ & $23.8 \pm 4.3$ & $1.2 \pm 0.8$ & $208.3 \pm 85.9$ & $28.2 \pm 13.3$ & $45.7 \pm 35.8$ & $95 \pm 73.3$ & $0.9 \pm 0.4$ \\
10 & $63 \pm 21.5$ & $81.8 \pm 0.5$ & $16.8 \pm 4.8$ & $1.5 \pm 1.3$ & $160 \pm 61.8$ & $33 \pm 21.2$ & $49.3 \pm 30$ & $54.6 \pm 49.7$ & $0.9 \pm 0.5$ \\
11 & $54.9 \pm 22.4$ & $4 \pm 0.1$ & $11.3 \pm 5.3$ & $1.6 \pm 1.3$ & $118.7 \pm 42.3$ & $36.3 \pm 19.2$ & $55.4 \pm 30$ & $35 \pm 31.6$ & $1 \pm 0.5$ \\
12 & $58.5 \pm 23.8$ & $3.4 \pm 0$ & $5.6 \pm 3.9$ & $1.4 \pm 0.8$ & $102.9 \pm 41.7$ & $42 \pm 20.9$ & $49.8 \pm 24.5$ & $28.2 \pm 25$ & $1.2 \pm 0.6$ \\
\hline
\end{tabular}


Table S4 The detailed contribution of each VOC group to the total $\mathrm{OH}$ reactivity during the sampling periods.

\begin{tabular}{|c|c|c|c|c|c|c|c|}
\hline Species & 2018 & Species & 2019 & Species & 2020 & Species & Average \\
\hline Isoprene & 1.7 & Isoprene & 1.1 & Isoprene & 1.1 & Isoprene & 1.8 \\
\hline Ethene & 1.4 & Ethene & 0.8 & Ethene & 0.9 & Ethene & 1.1 \\
\hline cis-2-Butene & 0.6 & Propene & 0.6 & $\mathrm{~m} / \mathrm{p}$-Xylene & 0.5 & Propene & 0.5 \\
\hline $\mathrm{m} / \mathrm{p}$-Xylene & 0.6 & $\mathrm{~m} / \mathrm{p}$-Xylene & 0.5 & Propene & 0.4 & $\mathrm{~m} / \mathrm{p}$-Xylene & 0.5 \\
\hline Propene & 0.6 & Styrene & 0.3 & Styrene & 0.4 & Styrene & 0.4 \\
\hline Styrene & 0.6 & trans-2-Butene & 0.3 & Toluene & 0.2 & cis-2-Butene & 0.3 \\
\hline trans-2-Butene & 0.4 & cis-2-Butene & 0.2 & trans-2-Butene & 0.2 & trans-2-Butene & 0.3 \\
\hline Toluene & 0.3 & Toluene & 0.2 & cis-2-Butene & 0.2 & Toluene & 0.3 \\
\hline i-Pentane & 0.2 & i-Pentane & 0.2 & 1-Butene & 0.1 & i-Pentane & 0.2 \\
\hline n-Pentane & 0.2 & 1-Butene & 0.2 & i-Pentane & 0.1 & 1-Butene & 0.2 \\
\hline Cyclopentane & 0.2 & trans-2-Pentene & 0.2 & n-Butane & 0.1 & n-Butane & 0.1 \\
\hline 1-Hexene & 0.2 & n-Butane & 0.2 & Propane & 0.1 & trans-2-Pentene & 0.1 \\
\hline $\begin{array}{c}\text { 1,3,5-Trimethy } \\
\text { lbenzene }\end{array}$ & 0.2 & Propane & 0.1 & trans-2-Pentene & 0.1 & Propane & 0.1 \\
\hline cis-2-Pentene & 0.2 & n-Pentane & 0.1 & o-Xylene & 0.1 & n-Pentane & 0.1 \\
\hline trans-2-Pentene & 0.2 & o-Xylene & 0.1 & $\begin{array}{c}\text { 1,2,4-Trimethylb } \\
\text { enzene }\end{array}$ & 0.1 & o-Xylene & 0.1 \\
\hline n-Butane & 0.2 & i-Butane & 0.1 & Ethylbenzene & 0.1 & $\begin{array}{l}\text { 1,3,5-Trimethylb } \\
\text { enzene }\end{array}$ & 0.1 \\
\hline 1-Butene & 0.1 & Ethylbenzene & 0.1 & i-Butane & 0.1 & cis-2-Pentene & 0.1 \\
\hline o-Xylene & 0.1 & $\begin{array}{l}\text { 3-Methylpenta } \\
\text { ne }\end{array}$ & 0.1 & $\begin{array}{c}\text { 1,3,5-Trimethylb } \\
\text { enzene }\end{array}$ & 0.1 & Ethylbenzene & 0.1 \\
\hline Propane & 0.1 & n-Hexane & 0.1 & n-Pentane & 0.1 & 1-Hexene & 0.1 \\
\hline Ethylbenzene & 0.1 & cis-2-Pentene & 0.1 & $\begin{array}{c}\text { 1,2,3-Trimethylb } \\
\text { enzene }\end{array}$ & 0.1 & Cyclopentane & 0.1 \\
\hline
\end{tabular}


Table S5 The detailed contribution of each VOC group to the total $\mathrm{OH}$ reactivity in different seasons.

\begin{tabular}{|c|c|c|c|c|c|c|c|}
\hline Species & Winter & Species & Spring & Species & Summer & Species & Autumn \\
\hline Ethene & 1.3 & Isoprene & 1.2 & Isoprene & 2 & Isoprene & 0.6 \\
\hline Propene & 1 & Ethene & 1.1 & $\mathrm{~m} / \mathrm{p}$-Xylene & 0.5 & Propene & 0.6 \\
\hline $\mathrm{m} / \mathrm{p}$-Xylene & 0.6 & Propene & 0.5 & Propene & 0.4 & $\mathrm{~m} / \mathrm{p}$-Xylene & 0.5 \\
\hline Isoprene & 0.5 & trans-2-Butene & 0.5 & Styrene & 0.3 & Styrene & 0.4 \\
\hline Styrene & 0.4 & m/p-Xylene & 0.4 & Ethene & 0.2 & Ethene & 0.4 \\
\hline cis-2-Butene & 0.3 & cis-2-Butene & 0.3 & cis-2-Butene & 0.2 & Toluene & 0.3 \\
\hline trans-2-Butene & 0.3 & Styrene & 0.2 & Toluene & 0.2 & trans-2-Butene & 0.2 \\
\hline Toluene & 0.3 & 1-Butene & 0.2 & trans-2-Butene & 0.2 & n-Butane & 0.2 \\
\hline 1-Butene & 0.3 & Toluene & 0.2 & i-Pentane & 0.2 & Propane & 0.2 \\
\hline i-Pentane & 0.2 & trans-2-Pentene & 0.2 & 1-Butene & 0.2 & i-Pentane & 0.2 \\
\hline trans-2-Pentene & 0.2 & i-Pentane & 0.2 & trans-2-Pentene & 0.1 & 1-Butene & 0.2 \\
\hline Propane & 0.2 & Acetylene & 0.1 & n-Butane & 0.1 & cis-2-Butene & 0.1 \\
\hline n-Butane & 0.2 & n-Butane & 0.1 & 3-Methylpentane & 0.1 & trans-2-Pentene & 0.1 \\
\hline n-Pentane & 0.1 & Propane & 0.1 & Ethylbenzene & 0.1 & $\begin{array}{c}\text { 1,3,5-Trimethylben } \\
\text { zene }\end{array}$ & 0.1 \\
\hline cis-2-Pentene & 0.1 & o-Xylene & 0.1 & o-Xylene & 0.1 & n-Pentane & 0.1 \\
\hline i-Butane & 0.1 & n-Pentane & 0.1 & Propane & 0.1 & i-Butane & 0.1 \\
\hline n-Hexane & 0.1 & 3-Methylpentane & 0.1 & m-Ethyltoluene & 0.1 & o-Xylene & 0.1 \\
\hline o-Xylene & 0.1 & cis-2-Pentene & 0.1 & n-Hexane & 0.1 & Ethylbenzene & 0.1 \\
\hline Ethane & 0.1 & n-Hexane & 0.1 & n-Pentane & 0.1 & Cyclopentane & 0.1 \\
\hline Acetylene & 0.1 & i-Butane & 0.1 & cis-2-Pentene & 0.1 & 3-Methylpentane & 0.1 \\
\hline
\end{tabular}




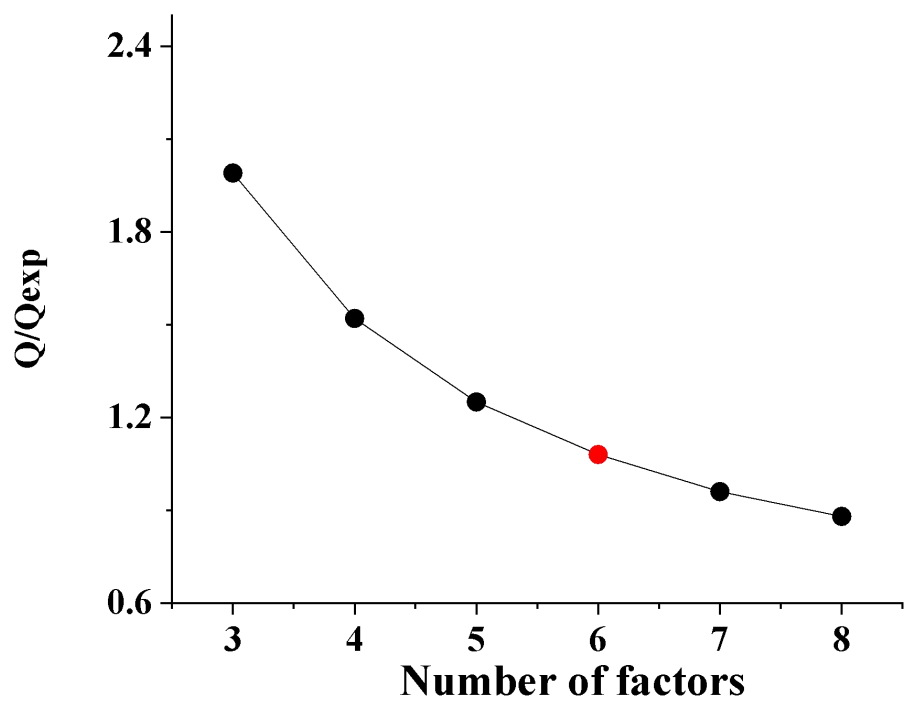

Fig. S1 The ratios of Q/Qexp at factor size ranged from 2-8. 


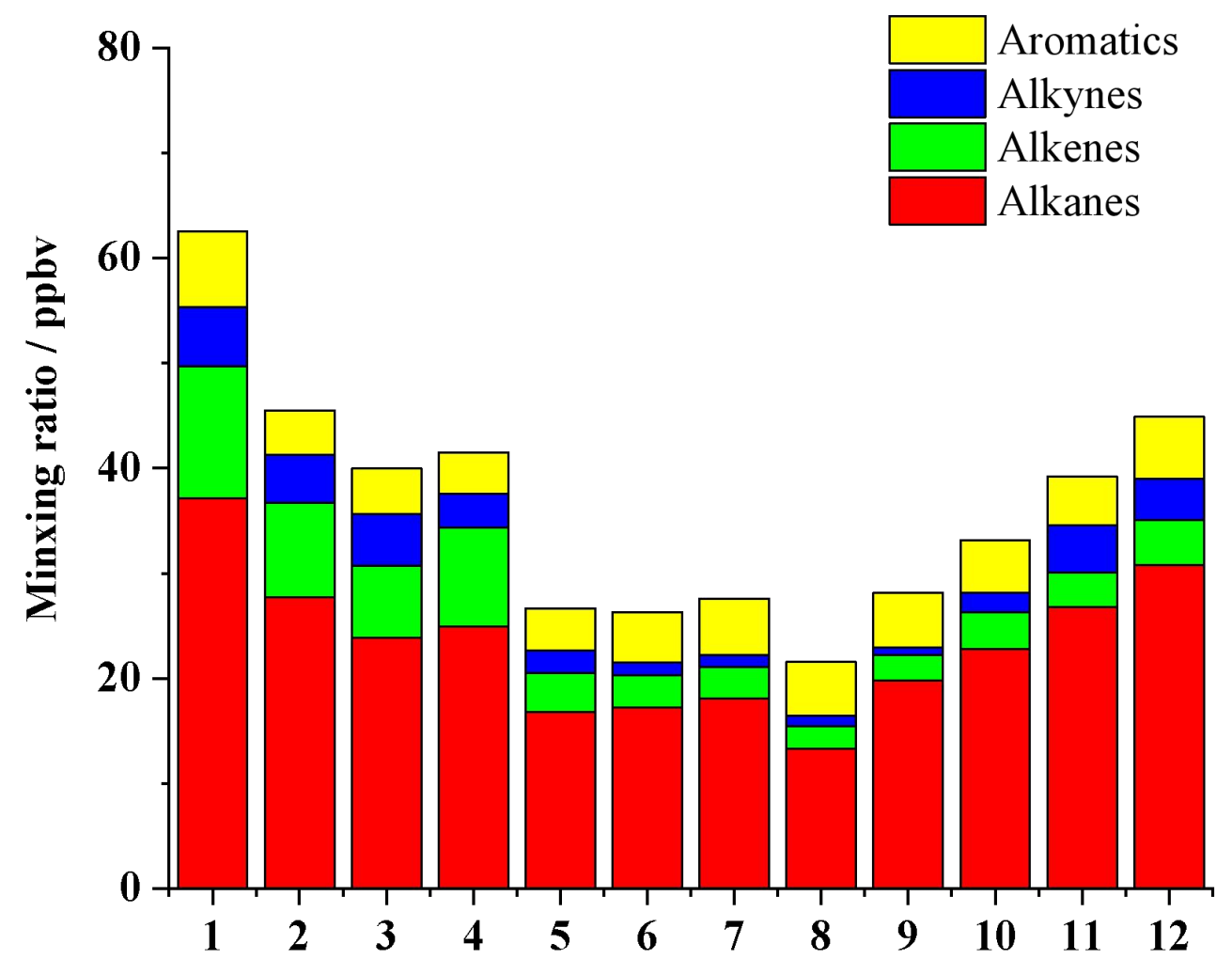

Fig. S2 Monthly changes in the concentrations of VOCs in Zhengzhou. 

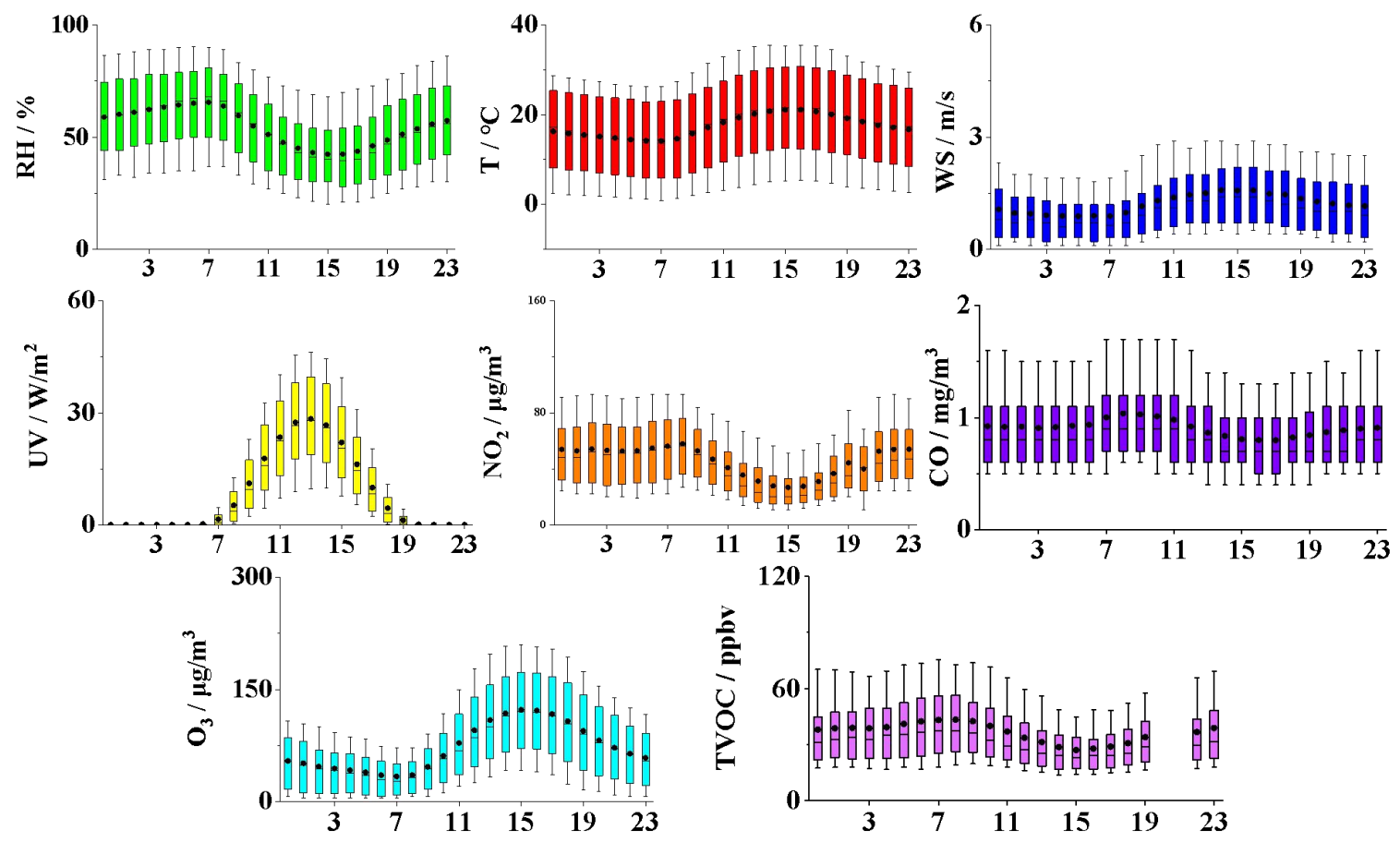

Fig. S3 Diurnal variations of VOCs meteorological conditions during the measurements. 

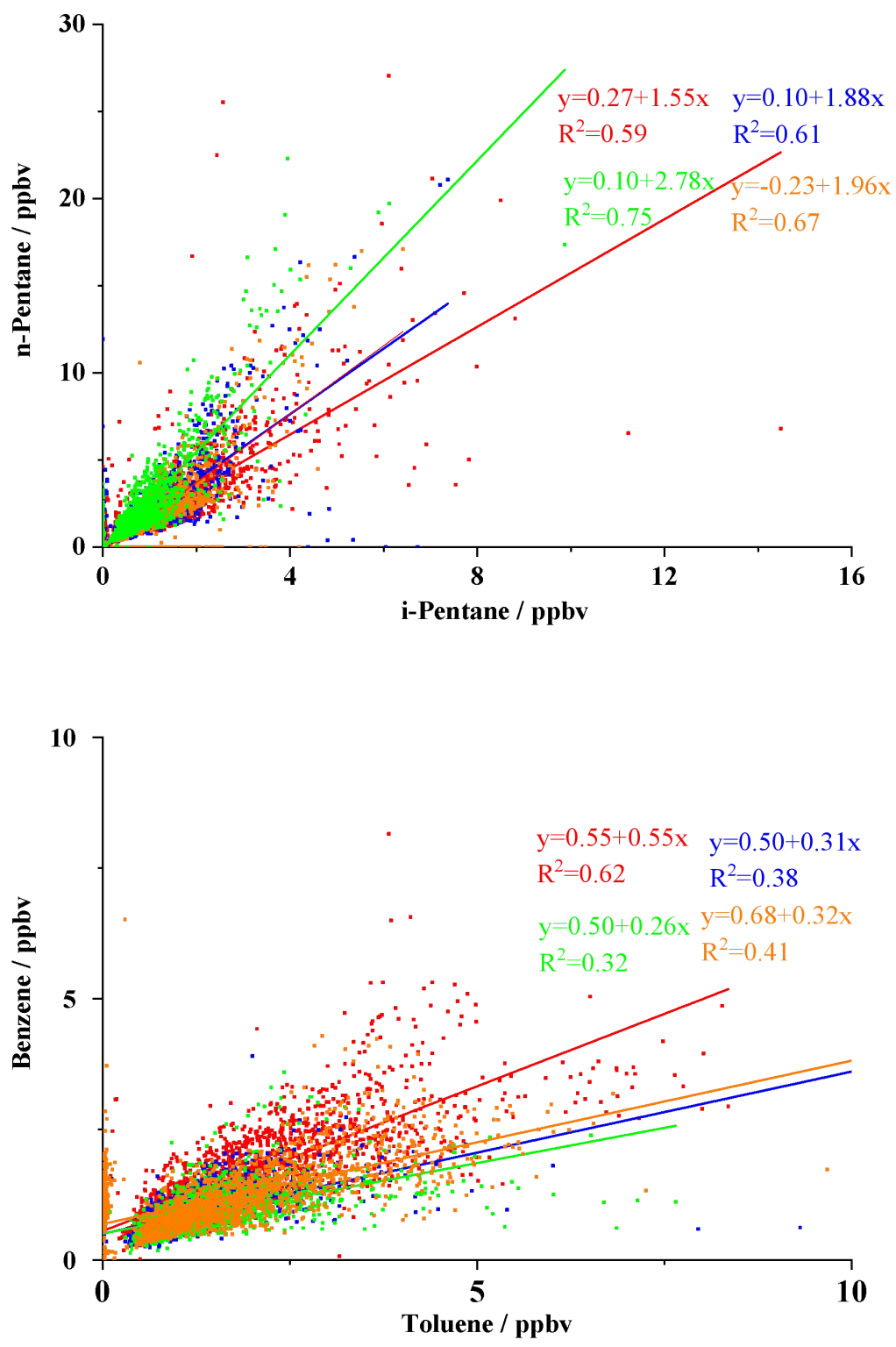

Fig. S4 Seasonal variation in $\mathrm{B} / \mathrm{T}$ and $\mathrm{i}-\mathrm{n}$-Pentane in Zhengzhou. 

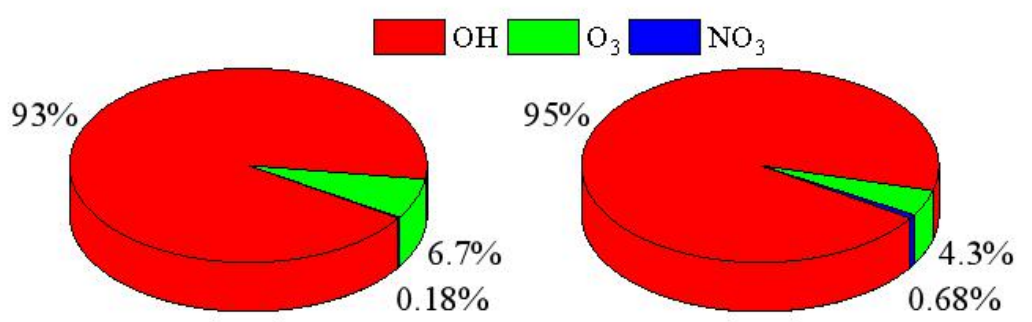

Spring: Total $\mathrm{AOC}=5.8 \times 10^{7}$ (molecules $\mathrm{cm}^{-3} \mathrm{~s}^{-1}$ )

Summer:Total $\mathrm{AOC}=7.5 \times 10^{7}$

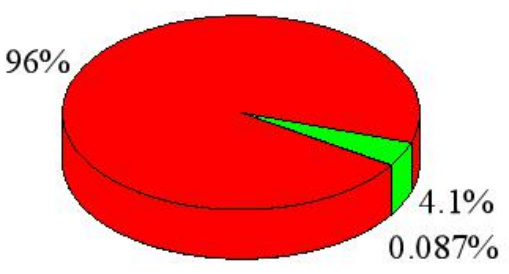
(molecules $\mathrm{cm}^{-3} \mathrm{~s}^{-1}$ )

Atumn:Total AOC $=5.8 \times 10^{7}$ (molecules $\mathrm{cm}^{-3} \mathrm{~s}^{-1}$ )

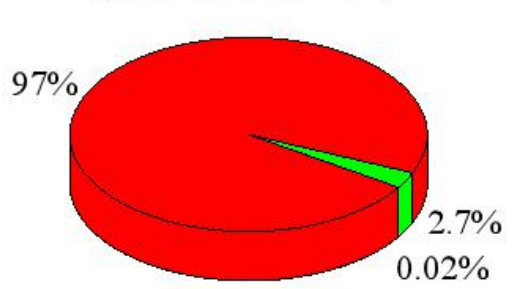

Winter:Total $\mathrm{AOC}=6.4 \times 10^{7}$ (molecules $\mathrm{cm}^{-3} \mathrm{~s}^{-1}$ )

Fig. S5 Comparison of the relative contributions of $\mathrm{OH}, \mathrm{O}_{3}$ and $\mathrm{NO}_{3}$ of the $\mathrm{AOC}$ in Zhengzhou in different seasons. 


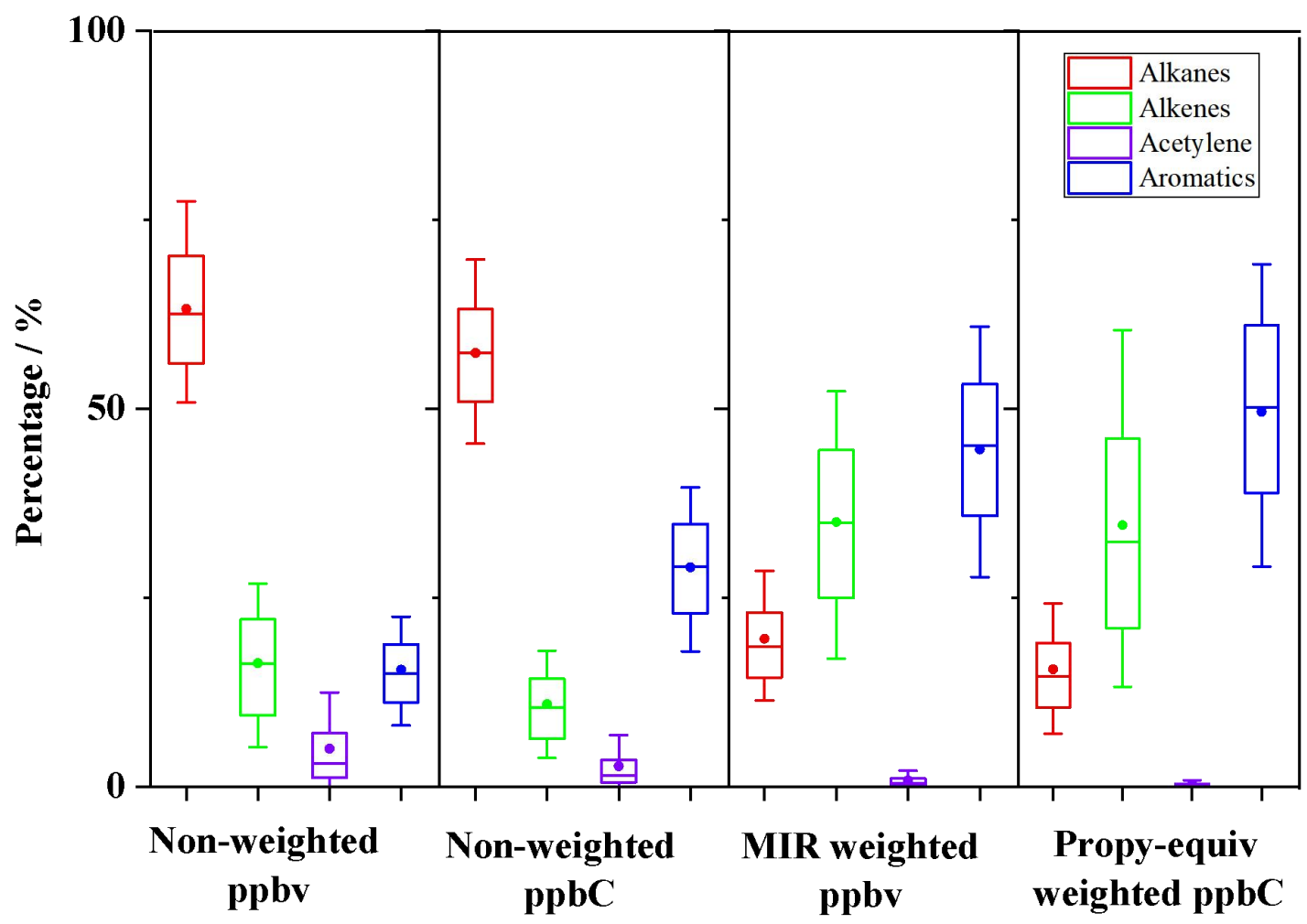

Fig. S6 Box and whisker plots of VOC profiles based on different scales during the entire sampling period. 


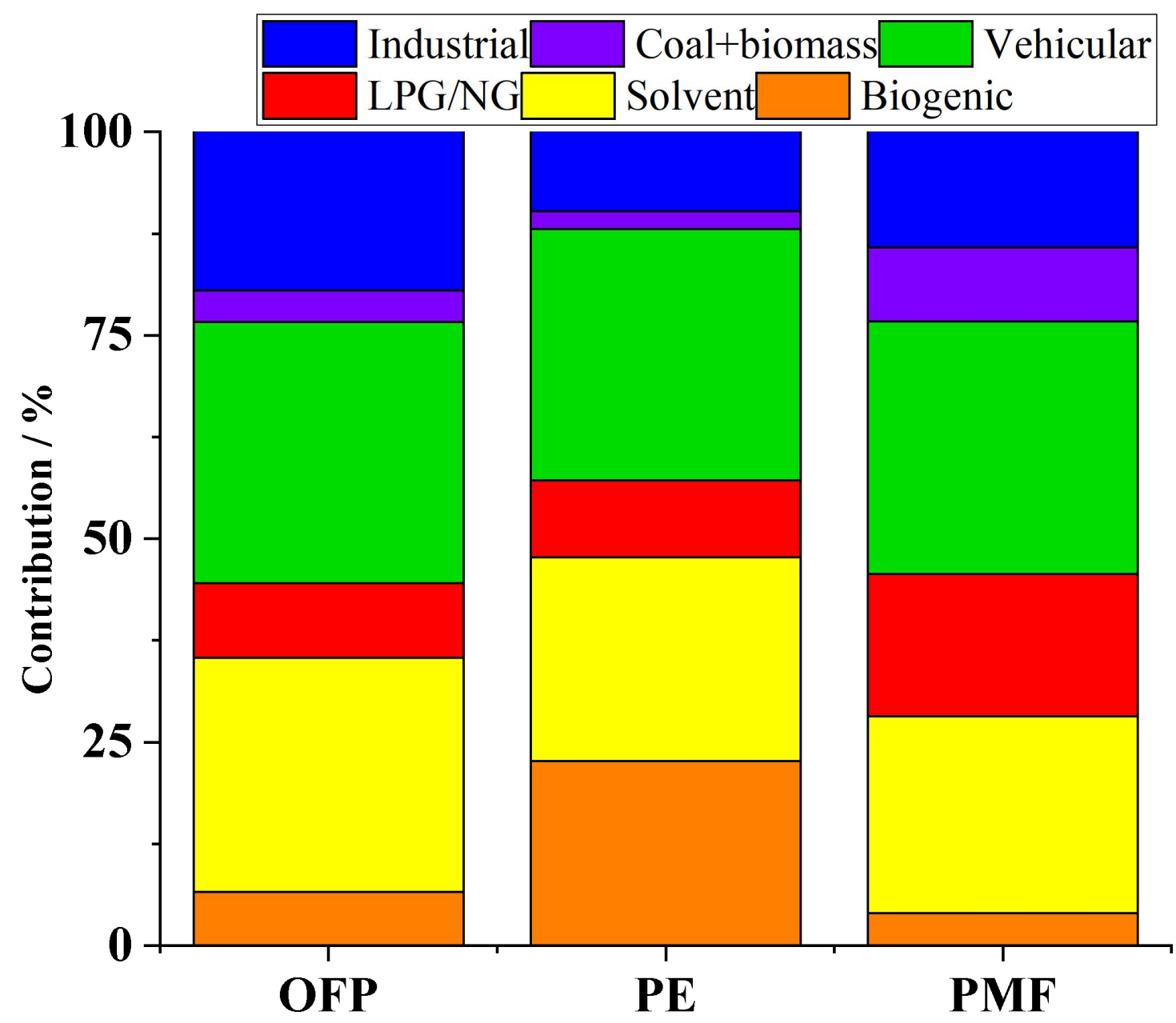

Fig.S7 Contribution of each source calculated using PMF, OFP, and PE. 


\section{References}

Atkinson, R., Arey, J., 2003. Atmospheric degradation of volatile organic compounds. Chem. Rev. 103, 4605-4638.

Carter, W., 1994. Development of ozone reactivity scales for volatile organic compounds. J. Air Waste Manag. Assoc. 44, 881-899.

Gao, J., Zhang, J., Li, H., Li, L., Xu, L., Zhang, Y., Wang, Z., Wang, X., Zhang, W., Chen, Y., Cheng, X., Zhang, H., Peng, L., Chai, F., Wei, Y., 2018. Comparative study of volatile organic compounds in ambient air using observed mixing ratios and initial mixing ratios taking chemical loss into account - A case study in a typical urban area in Beijing. Sci. Total Environ. 628-629, 791-804.

Norris, G., Duvall, R., Brown, S., Bai, S., 2014. EPA Positive Matrix Factorization (PMF) 5.0 Fundamentals and User Guide, U.S. Environmental Protection Agency National Exposure Research Laboratory Research Triangle Park, NC.

Song, C., Liu, B., Dai, Q., Li, H., Mao, H., 2019a. Temperature dependence and source apportionment of volatile organic compounds (VOCs) at an urban site on the north China plain. Atmos. Environ. 207, 167-181.

Song, M., Liu, X., Zhang, Y., Shao, M., Lu, K., Tan, Q., Feng, M., Qu, Y., 2019b. Sources and abatement mechanisms of VOCs in southern China. Atmos. Environ. $201,28-40$.

Yadav, R., Sahu, L., Tripathi, N., Pal, D., Beig, G., Jaaffrey, S., 2019. Investigation of emission characteristics of NMVOCs over urban site of western India. Environ. Pollut. 252, 245-255. 